\title{
CAN THE FUNDAMENTAL (HOMOTOPY) GROUP OF A SPACE BE THE RATIONALS?
}

\author{
SAHARON SHELAH
}

(Communicated by Haynes R. Miller)

\begin{abstract}
We prove that for any topological space which is metric, compact (hence separable) path connected and locally path connected, its homotopy group is not the additive group of the rational, moreover if it is not finitely generated then it has the cardinality of the continuum.
\end{abstract}

We prove here

1. THEOREM. Let $X$ be a compact metric (topological) space which is path connected and locally path connected. If the homotopy group of $X$ is not finitely generated then it has the power of the continuum (in fact there is a perfect set of nonhomotopic $f$ 's).

REMARK. $X$ is locally path connected if for every open $u$ and $y \in u$ there is a path connected $u^{\prime}, y \in u^{\prime} \subseteq u$. We shall really use only "weakly" locally path connected (see Definition 4).

2. INTRODUCTORY REMARKS. We have taken some trouble to make this accessible to both algebraic topologists and logicians (hence most other mathematicians), resulting in making the proof longer.

Nevertheless we assume e.g. that the reader understands what the theorem says. On the topological notions we use, see e.g. [Sp].

A conclusion of the theorem is that the additive group of the rationals cannot be the homotopy group of such a space.

The theorem answers a problem of Mycielski. I thank M. Foreman for asking me about it. On theorems related to Lemma 7 and their history see Harrington and Shelah [HS and Sh1] to which our exposition is closer.

The proof gives not only continuum many but a perfect set of paths (from say $x_{0}$ to $\left.x_{0}\right)$ nonhomotopic in pairs.

I thank H. Miller for improving the presentation.

3. Notation. $\mathbf{Z}$ is the set of integers, $\mathbf{Z}^{+}$the set of strictly positive integers. $\mathbf{R}$ is the set of reals, $\mathbf{R}^{+}$the set of strictly positive reals.

Let $I=[0,1]=\{t \in \mathbf{R}: 0 \leq t \leq 1\}$, endowed with the natural topology.

A path $f$ in a topological space $X$ is a continuous function from $I$ to $X$. We say $f$ is from $x$ to $y$ if $f(0)=x, f(1)=y$. We let $f^{-1}$ be defined by $f^{-1}(t)=f(1-t)$ (so it goes from $f(1)$ to $f(0)$ ). For paths $f, g, f g$ is defined only if $f(1)=g(0)$ and

Received by the editors June 22, 1985 and, in revised form, March 19, 1987.

1980 Mathematics Subject Classification (1985 Revision). Primary 55Q99; Secondary 03E15.

Research partially supported by the NSF and BSF. 
then

$$
f g(t)= \begin{cases}f(2 t), & 0 \leq t \leq 1 / 2 \\ g(2 t-1), & 1 / 2 \leq t \leq 1\end{cases}
$$

Let $E\left(x_{0}, X\right)=$ the family of paths from $x_{0}$ to $x_{0}$.

Let $[f]$ be the equivalence class of $f$ under the equivalence relation of being homotopic relative to the endpoints. Dividing by it, $E\left(x_{0}, X\right)$ becomes a group under the two operations defined above, which we call $\pi\left(X, x_{0}\right)$.

In a topological space a set is perfect if it is closed nonempty with no isolated point. For what we actually get, see 12 and the definition of $f_{A}$.

For a metric space, $d$ is the metric and $B_{r}(x)=B(x, r)=\{y \in X: d(y, x)<r\}$.

4. DEFINITION. Say that a space $X$ is graphical if it admits an open cover $\mathscr{U}$ such that for $u, v \in \mathscr{U}$, and all $x \in u, y \in v$, any two paths from $x$ to $y$ in $u \cup v$ are homotopic (relative to the end points) in $X$.

REMARK. For metric compact $X, X$ is graphical iff

(*) for every $x \in X$ for some open $u, x \in u$, and for every $y, z \in u$ any two paths from $x$ to $y$ in $u$ are homotopic (relative to end points) in $X$.

5. Definition. (1) Say that $X$ is weakly locally path connected (WLPC) if for every $x \in X$ and every neighborhood $u$ of $x$, there exists a neighborhood $v$ of $x$ in $U$ such that any point in $v$ can be joined to $x$ by a path through $u$.

6. DEFINITION. (2) Say that $X$ is semilocally simply connected (SLPC) if every point $x \in X$ has a neighborhood $u$ such that $\pi_{1}(u ; x) \rightarrow \pi_{1}(X ; x)$ is the trivial homomorphism (i.e. any two paths in $E(x, u)$ are homotopic in $X)$.

7. LEMMA. If $X$ is a compact graphical WLPC space then $\pi_{1}\left(X, x_{0}\right)$ is a finitely generated group for any $x_{0} \in X$.

PROOF. As $X$ is graphical, there is an open cover $\mathscr{U}$ of $X$ as in Definition 4.

For every $y \in X$ choose $u_{y}^{0} \in \mathscr{U}$ such that $y \in u_{y}^{0}$. As $X$ is WLPC for some open $u_{y}^{1}, y \in u_{y}^{1} \subseteq u_{y}^{0}$ and any point in $u_{y}^{1}$ is connected to $y$ by a path through $u_{y}^{0}$. So $X=\bigcup\left\{u_{y}^{1}: y \in X\right\}$ but $X$ is compact hence for some finite $Y \subseteq X$, $X=\bigcup\left\{u_{y}^{1}: y \in Y\right\}$, w.l.o.g. $x_{0} \in Y$.

For a path $f$ we say that $f$ is of type $\left\langle y_{0}, \ldots, y_{n}\right\rangle$ if there are reals $0=t_{0} \leq t_{1} \leq$ $\cdots \leq t_{n}=1$ exemplifying it, i.e. such that for $l=0, \ldots, n-1 \operatorname{Rang}\left(f \uparrow\left[t_{l}, t_{l+1}\right]\right) \subseteq$ $u_{y_{l}}^{0} \cup u_{y_{l+1}}^{0}$, and for $l=0, \ldots, n f\left(t_{l}\right) \in u_{y_{l}}^{1}$.

Note that one $f$ may have many types; however every $f$ has at least one type. (For every $t \in[0,1]$, for some $y \in Y, h(t) \in u_{y}^{1}$, hence for some open interval $J$ of $[0,1], t \in J$ and $f$ maps $J$ into $u_{y}^{1}$, and apply the compactness of $[0,1]$.)

$$
\text { If } f^{1}, f^{2} \in E\left(x_{0}, X\right) \text { both have type }\left\langle y_{0}, \ldots, y_{n-1}\right\rangle \text { then }
$$

$$
f^{1}, f^{2} \text { are homotopic. }
$$

We shall prove this later. Let

$$
\begin{aligned}
& F=\left\{[f]: f \in E\left(x_{0}, X\right) \text {, and for some } n \leq 2|Y|+8\right. \text { and } \\
& \left.\qquad y_{0}, \ldots, y_{n-1} \in Y, f \text { has type }\left\langle y_{0}, \ldots, y_{n-1}\right\rangle\right\} .
\end{aligned}
$$

By $(*) F$ (which is a subset of the fundamental group) has power $\leq$ $\sum_{i \leq 2|Y|+8}|Y|^{2 i}$ hence is finite. So it suffices to show that $F$ generates the fundamental group. 
So let $f \in E\left(x_{0}, X\right)$, and we shall prove $[f]$ is in the subgroup which $F$ generates. We know that for some $n$ and $\left\langle y_{0}, \ldots, y_{n-1}\right\rangle, f$ is of type $\left\langle y_{0}, \ldots, y_{n-1}\right\rangle$ and let $\left\langle t_{0}, \ldots, t_{n-1}\right\rangle$ exemplify it. We prove by induction on $n$. We prove that $[f]$ belongs to the subgroup generated by $F$. If $n \leq 2|Y|+8$ this is obvious. If not, let $i=$ $|Y|+4$. Let $\left\langle z_{0}, \ldots, z_{k}\right\rangle$ be a sequence of minimal length such that $\left\{z_{0}, \ldots, z_{k}\right\} \subseteq Y$, $z_{0}=x_{0}, z_{k}=y_{i}$ and for each $l \leq k-1, u_{z_{l}}^{1} \cap u_{z_{l+1}}^{1} \neq \varnothing$. Hence there is a path $h_{l}$ from $z_{l}$ to $z_{l+1}, \operatorname{Rang}\left(h_{l}\right) \subseteq u_{z_{l}}^{0} \cup u_{z_{l+1}}^{0}$. (Use paths from $z_{l}$ and $z_{l+1}$ to some point in $u_{z_{l}}^{1} \cap u_{z_{l+1}}^{1}$.) Also there is a path $h$ from $y_{i}=z_{k}$ to $f\left(t_{i}\right), \operatorname{Rang}(h) \subseteq u_{y_{i}}^{0}$ (as $f\left(t_{i}\right) \in u_{y_{i}}^{1}$ ). So $h_{0} h_{1} \cdots h_{k-1}$ is a path from $z_{0}=x_{0}$ to $z_{k}=y_{i}$ of type $\left\langle z_{0}, \ldots, z_{k-1}\right\rangle$.

Now let $f_{m}=f \uparrow\left[t_{m}, t_{m+1}\right]$ so

$$
\begin{gathered}
{[f]=\left[f_{0} f_{1} \cdots f_{i} \cdots f_{n-1}\right]=\left[f_{0}\right]\left[f_{1}\right] \cdots\left[f_{i}\right] \cdots\left[f_{n-1}\right]=\left[f_{0}\right]\left[f_{1}\right] \cdots} \\
{\left[f_{i-1}\right][h]^{-1}\left[h_{k-1}\right]^{-1} \cdots\left[h_{0}\right]^{-1}\left(\left[h_{0}\right] \cdots\left[h_{k-2}\right]\left[h_{k-1}\right][h]\right)\left[f_{i}\right]\left[f_{i+1}\right] \cdots} \\
{\left[f_{n-1}\right]=\left[f_{0} f_{1} \cdots f_{i-1} h^{-1} h_{k-1}^{-1} h_{k-2}^{-1} \cdots h_{0}^{-1}\right]\left[h_{0} h_{1} \cdots h_{k-1} h f_{i} f_{i+1} \cdots f_{n-1}\right] .}
\end{gathered}
$$

So $[f]$ is the product of two elements (from $\left\{\left[f^{\prime}\right]: f^{\prime} \in E\left(x_{0}, X\right)\right\}$. The first has naturally type $\left\langle y_{0}, \ldots, y_{i-1}, y_{i}, z_{k-1}, z_{k-2}, \ldots, z_{0}\right\rangle$, but $i=|Y|+4, k \leq|Y|$, so it belongs to $F$.

The second has naturally type $\left\langle z_{0}, \ldots, z_{k-2}, z_{k-1}, y_{i}, y_{i+1} \cdots y_{n-1}\right\rangle$ which has length $n-i+k$, but as $i=|Y|+4, k \leq|Y|$ this $\leq n-1$, so it belongs to $F$.

PROOF OF $(*)$. Let $\left\langle t_{0}^{l}, \ldots, t_{n}^{l}\right\rangle$ exemplify that $f^{l}$ has type $\left\langle y_{0}, \ldots, y_{n}\right\rangle$ for $l=1,2$. So $0=t_{0}^{l} \leq t_{1}^{l} \leq \cdots \leq t_{n-1}^{l} \leq t_{n}^{l}=1$ and $\operatorname{Rang}\left(f^{l} \mid\left[t_{m}^{l}, t_{m+1}^{l}\right]\right) \subseteq$ $u_{y_{m}}^{0} \cup u_{y_{m+1}}^{0}, f^{l}\left(t_{m}^{l}\right) \in u_{y_{m}}^{1}$.

For each $m=1, \ldots, n-1$ the points $f^{1}\left(t_{m}^{1}\right)$ and $f^{2}\left(t_{m}^{2}\right)$ are in $u_{y_{m}}^{1}$ so there is a path $g_{m}$ from $f^{1}\left(t_{m}^{1}\right)$ to $f^{2}\left(t_{m}^{2}\right)$ such that $\operatorname{Rang}\left(g_{m}\right) \subseteq u_{y_{m}}^{0}$. (Use paths from $f^{l}\left(t_{m}^{l}\right)$ to $y_{m}$ for $l=1,2$.)

Let $f_{m}^{l}=f^{l} \uparrow\left[t_{m}, t_{m+1}\right]$ for $m=0, \ldots, n-1$. Now

$$
\begin{aligned}
{\left[f^{1}\right] } & =\left[f_{0}^{1} f_{1}^{1} \cdots f_{n-1}^{1}\right]=\left[f_{0}^{1}\right]\left[f_{1}^{1}\right] \cdots\left[f_{n-1}^{1}\right] \\
& =\left(\left[f_{0}^{1}\right]\left[g_{1}\right]\right)\left(\left[g_{1}\right]^{-1}\left[f_{1}^{1}\right]\left[g_{2}\right]\right)\left(\left[g_{2}\right]^{-1}\left[f_{2}^{1}\right]\left[g_{2}\right]\right) \cdots\left(\left[g_{n-1}\right]^{-1}\left[f_{n-1}^{1}\right]\right),
\end{aligned}
$$

and

$$
\left[f^{2}\right]=\left[f_{0}^{2} f_{1}^{2} \cdots f_{n-1}^{2}\right]=\left[f_{0}^{2}\right]\left[f_{1}^{2}\right] \cdots\left[f_{n-1}^{2}\right] .
$$

So for proving equality it is enough to show:

(a) $\left[f_{0}^{1} g_{1}\right]=\left[f_{0}^{2}\right]$

(b) $\left[g_{m}^{-1} f_{m}^{1} g_{m+1}\right]=\left[f_{m}^{2}\right]$,

(c) $\left[g_{m-1}^{-1} f_{n-1}^{1}\right]=\left[f_{n-1}^{2}\right]$.

Now (b) holds as $g_{m}^{-1} f_{m}^{1} g_{m+1},\left(f_{m}^{2}\right)$ are homotopic by the choice of $\mathscr{U}$ and as $u_{y_{m}}^{0}, u_{y_{m+1}}^{0} \in \mathscr{U}$. For (a), (c) the situation is similar.

8. LEMMA. A metric space is graphical provided it is WLPC and SLPC.

ProOF. For each $x \in X$, let $\varepsilon(x)>0$ be such that $\pi_{1}\left(B_{\varepsilon(x)}(x), x\right) \rightarrow \pi_{1}(X, x)$ is trivial (exists as $X$ is SLPC). Let $\delta(x)>0$ be such that any point in $B_{\delta(x)}(x)$ is connected to $x$ by a path in $B_{\varepsilon(x) / 3}(x)$ (exists as $X$ is WLPC). Then $\left\{B_{\delta(x)}(x): x \in\right.$ $X\}$ is an open cover of $X$. Pick a pair of points, $x_{1}$ and $x_{2}$ and let $B_{i}=B_{\delta\left(x_{i}\right)}\left(x_{i}\right)$. Let $y_{i} \in B_{i}, i=1,2$, and let $\alpha$ and $\beta$ be paths from $y_{1}$ to $y_{2}$ in $B_{1} \cup B_{2}$. Let $\gamma_{i}$ 
join $x_{i}$ to $y_{i}$ in $B_{\varepsilon\left(x_{i}\right) / 3}\left(x_{i}\right), i=1,2$. Then $\gamma_{1} \alpha \gamma_{2}^{-1}$ and $\gamma_{1} \beta \gamma_{2}^{-1}$ are paths from $x_{1}$ to $x_{2}$ in $C \stackrel{\text { def }}{=} B_{\varepsilon\left(x_{1}\right) / 3}\left(x_{1}\right) \cup B_{\varepsilon\left(x_{2}\right) / 3}\left(x_{2}\right)$. We may assume $\varepsilon\left(x_{1}\right) \geq \varepsilon\left(x_{2}\right)$. The triangle inequality implies then that $C \subseteq B_{\varepsilon\left(x_{1}\right)}\left(x_{1}\right)$, so $\left(\gamma_{1} \alpha \gamma_{2}^{-1}\right)\left(\gamma_{1} \beta \gamma_{2}^{-1}\right)^{-1} \simeq$ $\gamma_{1} \alpha \beta^{-1} \gamma_{2}^{-1}$ is null, and consequently $\alpha$ is homotopic (relative to endpoints) to $\beta$. Q.E.D

9. COROLLARY. If $X$ is a WLPC compact metric space and $\pi_{1}(X)$ is not finitely generated, then there exists a point $x \in X$ such that every neighborhood $U$ of $x$ contains a loop at $x$ (i.e. an $f \in \pi(X, x)$ ) which is essential in $X$ (i.e. $[f]$ is not the unit in the homotopy group).

So let $x_{0}, f_{n}\left(n \in \mathbf{Z}^{+}\right)$be such that $x_{0}$ is as in conclusion $8, f_{n} \in E\left(x_{0}, X\right)$, Rang $f_{n} \subseteq B_{1 / n}\left(x_{0}\right),\left[f_{n}\right]$ is not the unit in the homotopy group.

10. PROOF OF THEOREM 1. Fix $0=t_{0}<t_{1}<t_{2}<\cdots<1$ s.t. $1=\lim _{n \rightarrow \infty} t_{n}$ (so $0<t_{n}<t_{n+1}<1$ for $n \in \mathbf{Z}^{+}$) and for $A \subset \mathbf{Z}^{+}$put

$$
f_{A}(t)= \begin{cases}f_{n}\left(\left(t-t_{n-1}\right) /\left(t_{n}-t_{n-1}\right)\right) & \text { if } n \in A, t \in\left[t_{n-1}, t_{n}\right], \\ x_{0} & \text { if } n \notin A, t \in\left[t_{n-1}, t_{n}\right],\end{cases}
$$

So $f_{A}$ is in a sense the infinitary product $\prod_{n \in A} f_{n}$. By the choice of the $f_{n}$ 's, $f_{A}$ is continuous. Hence it represents a member of the homotopy group of $X$. Also $f_{\{n\}}$ represents the same element as $f_{n}$ and

$$
\text { if } n \text { is the first member of } A, B=A-\{n\} \text { then } f_{A} \text { and }
$$

$f_{n} f_{B}$ are homotopic.

11. FACT. If $A, B \subseteq \mathbf{Z}^{+}, n \notin B, A=B \cup\{n\}$ then $f_{A}, f_{B}$ are not homotopic.

PROOF OF 11. We prove it by induction on the number of $m<n$ which are in $B$. If this number is zero but $f_{A}, f_{B}$ are homotopic then by $(*)$ above " $f_{A}$ and $f_{n} f_{B}$ are homotopic" we get that $f_{n}$ is homotopic to zero, a contradiction to its choice.

Now Theorem 1 will follow immediately by Claim 12 and Lemma 13 below.

We define a relation $\mathscr{E}$ on $\Gamma=\left\{A: A\right.$ a subset of $\left.\mathbf{Z}^{+}\right\}$by $A \mathscr{E} B$ iff the mappings $f_{A}, f_{B}$ are homotopic.

12. Claim. $\mathscr{E}$ is an analytic (see below) equivalence relation.

12A. REMARK. (1) $\mathscr{E}$ is analytic means that the set $\{(A, B): A, B \in \Gamma, A \in B\}$ is an analytic subset of the product space $\Gamma \times \Gamma$.

(2) $\Delta \subset \Gamma$ is analytic if for some complete separable metric space $Y$ and Borel subset $P$ of $Y \times \Gamma$

$$
\Delta=\{A \in \Gamma: \text { for some } y \in Y,(y, A) \in P\} .
$$

We can replace "Borel" by "closed".

(3) Of course $\Gamma$ is endowed with the Tychonov topology (e.g. use the metric $d(A, B)=\operatorname{Inf}\left\{1 / 2^{n}: A \cap\{1, \ldots, n\}=B \cap\{1, \ldots, n\}\right\}$.

PROOF OF 12. By the basic properties of homotopy (see e.g. [Sp]) $\mathscr{E}$ is an equivalence relation. It is also true that $\mathscr{E}$ is an analytic relation. As $X$ is a compact and metric it is necessarily separable. So let $\left\{z_{n}: n \in \mathbf{Z}^{+}\right\} \subseteq X$ be a dense subset of $X$. Suppose $f_{A}, f_{B}$ are homotopic, then there is a continuous function $g$ from $[0,1] \times[0,1]$ into $X$, such that for every real $r g(r, 0)=f_{A}(r)$, $g(r, 1)=f_{B}(r)$. [More formally we should have written $f((r, 0)), f((r, 1))$.] We say 
in such cases that " $g$ exemplifies the homotopy of $f_{A}$ and $f_{B}$ ". As $[0,1] \times[0,1]$ is compact, $g$ is uniformly continuous, so there is a function $h: \mathbf{Z}^{+} \rightarrow \mathbf{Z}^{+}$such that

$$
\begin{aligned}
& m \in \mathbf{Z}^{+},\left(x_{1}, y_{1}\right),\left(x_{2}, y_{2}\right) \in[0,1] \times[0,1],\left|x_{1}-x_{2}\right|+\left|y_{i}-y_{i}\right| \\
& <1 / h(m) \text { implies } d\left(g\left(x_{1}, y_{1}\right), g\left(x_{2}, y_{2}\right)\right)<1 / m .
\end{aligned}
$$

We can code $g$ by a set $C_{g}$ of octuples of positive natural number (i.e. members of $\mathbf{Z}^{+}$),

$$
\begin{array}{r}
C_{g}=\left\{\left\langle l_{1}, k_{1}, l_{2}, k_{2}, n, m, i, j\right\rangle: l_{1}, k_{1}, l_{2}, k_{2}: n, m, i, j \text { belongs to } \mathbf{Z}^{+},\right. \\
\left.d\left(g\left(l_{1} / k_{1}, l_{2} / k_{2}\right), z_{n}\right)<n / m \text { and } h(i)=j\right\} .
\end{array}
$$

Let $P=\left\{\left\langle A, B, C_{g}\right\rangle: g\right.$ exemplifies the homotopy of $\left.f_{A}, f_{B}\right\}$ and $\Gamma_{8}=\{A: A \subseteq$ $\left.\left(\mathbf{Z}^{+}\right)^{8}\right\}$ again with the Tichonov topology. It is clear that

(1) $C_{g}$ determine $g$ (i.e. for every $C \subseteq\left(\mathbf{Z}^{+}\right)^{8}$ for at most one $g$ as above, $\left.C_{g}=C\right)$.

(2) $\Gamma_{8}$ is a separable metrizable space.

(3) $P$ is a Borel subset of $\Gamma_{8} \times \Gamma \times \Gamma$.

(4) $f_{A}, f_{B}$ are homotopic iff for some $C,\langle A, B, C\rangle \in P$.

By (3) and (4) and definition of analytic, we finish.

13. LEMMA. If $\mathscr{E}$ is an analytic equivalence relation on $\Gamma=\left\{A: A \subseteq \mathbf{Z}^{+}\right\}$ which satisfies

(*) if $A, B \subset \mathbf{Z}^{+}, n \notin B, A=B \cup\{n\}$ then $A, B$ are not $\mathscr{E}$-equivalent, then there is a perfect subset of $\Gamma$ of pairwise nonequivalent $A \subseteq \mathbf{Z}^{+}$.

REMARK. The proof uses some knowledge of set theory.

PROOF. Let $N$ be a countable elementary submodel of $\left(H\left(\left(2^{\aleph_{0}}\right)^{+}\right), \mathscr{E}\right)$ to which the real parameter in the definition of $\mathscr{E}$ belongs. Now

$(* *)$ if $\left\langle A_{1}, A_{2}\right\rangle$ be a pair of subsets of $\mathbf{Z}^{+}$which is Cohen generic over $N$ [this means that it belongs to no first category subset of $\Gamma \times \Gamma$ which belongs to $N]$ then

(1) $A_{1}, A_{2}$ are $\mathscr{E}$-equivalent in $N\left[A_{1}, A_{2}\right]$ if they are $\mathscr{E}$-equivalent.

(2) $A_{1}, A_{2}$ are non- $\mathscr{E}$-equivalent in $N\left[A_{1}, A_{2}\right]$.

PROOF OF (**). (1) By the absoluteness criterions.

(2) If not then some finite information forces this, hence for some $n$

(a) If $\left\langle A_{1}^{\prime}, A_{2}^{\prime}\right\rangle$ is Cohen generic over $N$ and $A_{1}^{\prime} \cap\{1, \ldots, n\}=A_{2} \cap\{1, \ldots, n\}$ and $A_{2}^{\prime} \cap\{1, \ldots, n\}=A_{2} \cap\{1, \ldots, n\}$ then $A_{1}^{\prime}, A_{2}^{\prime}$ are $\mathscr{E}$-equivalent in $N\left[A_{1}^{\prime}, A_{2}^{\prime}\right]$.

Let $A_{1}^{\prime \prime}$ be $A_{1} \cup\{n+1\}$ if $(n+1) \notin A_{1}$ and $A_{1}-\{n+1\}$ if $(n+1) \in A_{1}$.

Trivially also $\left\langle A_{1}^{\prime \prime}, A_{2}\right\rangle$ is Cohen generic over $N$, hence by $(\alpha)$ above $A_{1}^{\prime \prime}, A_{2}$ are $\mathscr{E}$-equivalent in $N\left[A_{1}^{\prime \prime}, A_{2}\right]$. By $(* *)(1)$ we know that really $A_{1}^{\prime \prime}, A_{2}$ are $\mathscr{E}$ equivalent. As equivalence is a transitive relation clearly $A_{1}, A_{1}^{\prime \prime}$ are $\mathscr{E}$-equivalent. But this contradicts the hypothesis $(*)$.

END OF THE PROOF OF THEOREM 1 . We can easily find a perfect (nonempty) subset $P$ of $\left\{A: A \subseteq \mathrm{Z}^{+}\right\}$such that for any distinct $A, B \in P,(A, B)$ is Cohen generic over $N$. So for $A, B \in P, N[A, B] \models$ " $A, B$ are not $\mathscr{E}$-equivalent" and by (**) (1) $A, B$ are not $\mathscr{E}$-equivalent. This finishes the proof of 13 hence of Theorem 1. We can similarly [HSh, Sh1] prove 
14. LEMMA. Suppose $\mathscr{E}$ is a co- $\kappa$-Souslin equivalence relation on some $\kappa$ Souslin set $\Gamma_{1} \subseteq P=\left\{A: A \subseteq \mathbf{Z}^{+}\right\}$, or $\Gamma_{1} \subseteq\left(\mathbf{Z}^{+}\right)^{\left(\mathbf{Z}^{+}\right)}=\{h: h$ a function from $\mathbf{Z}^{+}$to $\left.\mathbf{Z}^{+}\right\}$(equivalently ${ }^{\omega} \omega$ ) and $\Gamma_{1}$ has cardinality $>\kappa$. Suppose further

for every $n \in \mathbf{Z}^{+}$and function $h_{0}$ from $\mathbf{Z}^{+}$into $\mathbf{Z}^{+}$, there are

(*) functions $h_{1}, h_{2}: \mathbf{Z}^{+} \rightarrow \mathbf{Z}^{+}, \bigwedge_{i=1}^{n} h_{0}(i)=h_{1}(i)=h_{2}(i)$, not $\mathscr{E}$ equivalent but $\left\{m \in \mathbf{Z}^{+}: h_{0}(m) \neq h_{l}(m)\right\}$ is finite for $l=1,2$.

If we add a generic Cohen real $r$ to our universe $V$, in $V[r] \mathscr{E}$ is still an equivalence relation (i.e. its definition defines one).

Then there is a perfect family of sets $A \subseteq \mathbf{Z}^{+}$, pairwise non- $\mathscr{E}$-equivalent.

\section{REFERENCES}

[HSh] L. Harrington and S. Shelah, Counting equivalence classes for co- $\kappa$-Souslin relation, Proc. Conf. Prague, 1980 (D. van Dalen, D. Lascar and T. J. Smiley, eds.), Logic Colloq., NorthHolland, 1982, pp. 147-152.

[O1] P. Olum, Non-abelian cohomology and Van Campen's Theorem, Ann. of Math. (2) 68 (1958), $658-668$.

[Sh] S. Shelah, Can the fundamental group of a nice space be equivalent the rationals? Abstracts Amer. Math. Soc. 5 (1984), 217.

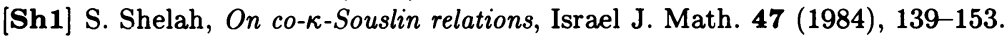

[Sp] E. H. Spanier, Algebraic topology, McGraw-Hill, 1966.

[V] E. Van Kampen, On the connection between the fundamental groups of some related spaces, Amer. J. Math. 55 (1933), 261-267.

Mathematics INSTitute, Hebrew UNiVersity, Jerusalem, ISRAEL 48109

Department of Mathematics, University of Michigan, Ann Arbor, Michigan

Department of Mathematics, Rutgers University, New Brunswick, NeW JerSEY 08903 